\title{
Methods Used to Eliminate the Students' Chintonglish Sentences in Their Writing
}

\author{
Xiaoying Zhou \\ School of Foreign Languages, Nanchang Normal University, Nanchang, China \\ Hangjie Liao \\ Purdue University, West Lafayette, USA
}

\begin{abstract}
In this paper the authors discuss four types of English in Chinese students' English writing. They are Pure English, China English, Sinicized English and Chinese Tone English (Chintonglish). As the Chintonglish (Chinese Tone English) sentences appear frequently in students writing, the authors will discuss this type of "English" from the psychological and cultural point of view, which may serve as a guide for the Chinese students' writing of good English. In the authors' opinion the methods used for the elimination of such Chintonglish sentences in the students' English writing are to point out the cause of such Chintonglish mistakes to the students clearly, create an English communicative environment and choose some typical pieces of passages for the students to read and imitate.
\end{abstract}

Index Terms —Chintonglish, English writing, psychology, elimination, methods, sentences

\section{INTRODUCTION}

Nowadays English writing is gradually becoming one of the required courses among Chinese students. In order to test students' ability writing is an important part in the popular national CET for students majoring in science or in English. The popular CET test has a section for English writing. That is why over the past few years many major language magazines carried many papers discussing English writing of college students.

However it's a pity that no matter how experienced an English teacher may be, Chinese students will inevitably hand in their written homework in Chinese Tone English, which is quite different from China English and is different from Sinicezed English, too. This kind of English is such a piece of writing, which appears like English but reads quite like Chinese. In other words, this kind of English is not English in its real sense. It is a piece of writing, which is just a collection of English words only sometimes here and there with some idiomatic expressions. It is just like a Chinese actor dressed in foreign costume. It appears like English but reads like Chinese. Therefore the authors call this kind of English as Chinese Tone English. As this kind of "English" occurs among Chinese college students writing quite frequently, the authors think it is high time to have a deep-going research in this kind of "English" so that English teachers may have a scientific view of this kind of "English" and can teach the students in a more scientific way and the students, in turn, can have a clear view of this kind of linguistic "symptom" and consciously avoid writing this "English" an their written homework. For the sake of convenience, the authors use in invented character "Chintonglish" to call this kind of "English", meaning "Chinese Tone English"

This type of "English" is quite different from China English and Sinicized English in many respects. Sinicized English refers to that type of English which is used by Chinese students or Chinese English learners. It is much influenced or interfered by their tongue. It is a deformed English which is not in conformity with English rules and regulations, neither is it in conformity with English cultural customs as well. As it is a type of deformed English, which will eventually be extinguished with the deepening of East-West communication. However someone holds that there is no clear distinction between China English and Sinicized English. It is just a matter of degree. Anybody who lives in China or has lived in China for a rather long period of time will surely be influenced by Chinese culture and consequently he must surely show their Sinicized traces in his writing or speaking. This is an objective law and nobody can change it. It involves many aspects, such as in the language itself, or the speaker's mental and cultural levels and so on. A Chinese will say: "Where? Where?" used to mean: "I'm flattered!" Because "Where? Where?" is a typical Chinese expression. If it is explained according to its surface meaning the listeners would become perplexed or even become confused without explanations. When the students translate “老郭” into English, they will often write as “old Guo" or "Lao Guo". It is a pity that both these two English forms are not correct, for here “老" means not being old in age but has some intimacy or informality meaning. It is often used between friends. Sometimes the addresser may be younger than the addressee. Therefore using "Old Guo!" or "Lao Guo!" are both incorrect from the view point of pure English. The most interesting phenomenon is "surname + lao" pattern (姓十老). For example we can not say or write “Guo Old” to express “郭老”. It is quite different from the original meaning. For “郭老”refers not only to a man famous for his knowledge, but also noted for his noble characters or something like that. This is a very popular 
expression in Chinese culture. In modern Chinese history there were three "Laos", they are Guo Mojo, Dong Biwu, Xu Teli etc. because they have all rendered their great contributions to Chinese society and consequently they won the Chinese people's respect and love. Here, an Old can never be used to express this meaning. .It is why someone uses "Venerable + surname" pattern to explain the meaning of this kind of expression. Thus Guo Mojo is called as Guo the venerable or "Venerable Guo" and so on and so forth. (Li Wenzhong 1993). As China is a country with very long history and rich cultural tradition, there must be a lot of pure Chinese expressions in Chinese language. Therefore, no matter how experienced or well informed a man may be he will not be able to avoid such expressions in the English writings. The question is how to minimize such kind of Sinicized English traces to the least.

However there is also one other type of English, which is not only different from China English, but also different from Sinicized English as well. In the authors' opinion it can be classified as a type of "Chinese Tone English". Chinese Tone English is a type of "English" most frequently used in Chinese students" writings. For the sake of convenience, the author uses in invented character "Chintonglish" to call this kind of "English".

\section{Methods Used to Eliminate the Students' Chintonglish SENTEnCES In Their Writing}

In the authors' opinion the methods used for the elimination of such Chintonglish sentences in the students' English writing are to point out the cause of such Chintonglish mistakes to the students clearly, to create an English communicative environment and to choose some typical pieces of passages for the students to read and imitate.

(1) Point out the cause of such Chintonglish mistakes to the students clearly

First of all the teachers must point out where lies the mistake in front of all the students so that the students may try their best to avoid such mistakes as much as possible. Checking their homework without explaining them the causes of their mistakes the result may not be obvious. For if such sentences in one piece of their English writing are corrected by the teacher, such mistakes may appear in another piece of writing again. For the influence of their mother tongue will follow them like a shadow whatever they are doing. It is only when the students are clearly aware of the cause of such mistakes can they consciously avoid such mistakes in their English writing as much as possible. So the teacher must point out where lies the mistake in front of all the students so that the students may try their best to avoid such mistakes as much as possible.

Generally speaking, there exist four types of English in writing among Chinese students. They are Pure English, China English, Sinicized English and Chinese Tone English (Chintonglish). Pure English is the standard English, which is not only true western in its content, but also quite English in its sentence grammar and text structure.. Besides, it reads quite English as well, for every sentence is carefully written and every word is carefully chosen. However it's a pity that the students who can write this type of English are quite rare in number. China English is based on the standard English as its core to express various things in Chinese culture. It is not influenced or interrupted by their mother tongue (Chinese) and enters into communication through such reproductive means as transliteration, borrowing and semantic reproduction. It is mainly composed of vocabulary, sentence patterns and text structure with Chinese nature. However, it should not be confused with Sinicized English, for it is something like British English. American English and Australia English, which bear some special meaning as compared with standard English. Sinicized English refers to that type of English which is used by Chinese students or Chinese English learners. It is much influenced or interfered by their tongue. It is a deformed English which is not in conformity with English rules and regulations, neither is it in conformity with English cultural customs as well.

However there is also one other type of English, which is not only different from China English, but also different from Sinicized English as well. In the authors' opinion it can be classified as a type of "Chinese Tone English". Owing to Chinese students' mental activities in writing English according to Chinese language, the sentences are not in conformity with English grammar rules. Therefore the author calls this kind of "English" as "Chinese Tone English"

(A) Chinese Tone English "Chintonglish"

As nowadays English writing is becoming an increasingly important part in Chinese English courses, and consequently English writing is becoming an important part in their English texts too. In view of this case the authors collected many such examples in the writing course. Upon analyses the author finds it very interesting to analyses such Chinese Tone English "Chintonglish" writings from the view point of psychology or social culture. For example in the teaching of English writing, the author once asked the students to write a letter to a friend of theirs. While checking their written homework the author came upon such a sentence "I have received your letter for three weeks". There is no doubt that the reader can know its meaning. But upon thinking carefully the authors find this sentence somewhat incorrect. How come? The authors find that this sentence is written out of Chinese grammar. For, while the student is writing this sentence, his mind naturally runs along the grammar structure of his native language --- Chinese without thinking whether the sentence he is writing is correct or not in its English grammar. In the hands of a foreigner this sentence must be written as "Your letter reached me three weeks ago" or "I received your letter three weeks ago", or even "I have delayed your letter for three weeks". Chinese Tone English "Chintonglish" is a type of "English" most frequently used in Chinese students" writing. In the teaching practice the authors collected a lot of such "Chinese Tone English" sentences. Through careful study the authors find that although these sentences seem quite divergent in their structures and meanings, they actually came out of the same stereotyped thinking pattern ---writing English out of Chinese grammar structure. No matter what he is writing, this pattern functions from the very beginning. Having this in 
mind the authors find that the very seemingly complicated English writing problem suddenly becomes quite simple and easy.

(B) Chinese Tone English "Chintonglish" sentences can be subdivided into four types:

Roughly speaking, these types of English sentences can be subdivided into the following types. (a): Logical confusion. (b): Structure confusion. (c): Incorrect use of words. (d): Grammatically wrong in the whole sentence.

\section{(a): Logical confusion}

From the view point of English language there must be some logically incorrect sentences while the students write their English sentences they think in Chinese grammar. Below this title are included a group of sentences, which are not logically correct. For examples once the authors asked the students to write a short article to compare the temperature of two cities in China. For these two cities are located far apart, one in north China and the other in the south. The author came upon the following sentences: "The temperature difference of the two cities is different," means "The two cities differ greatly in their temperature".; The most interesting sentence is the following one, which deals with the comparison of the liquid content in two bottles: "The liquid in C is more than two times B" (Meaning: The liquid in Bottle C is two times more than that in Bottle B); "Only know that there is six liters liquid in Bottle D" (Meaning: We only know that Bottle D contains six liters of liquid). There are many other such sentences, the above are just some of them. They are some logically incorrect sentences while the students write their English sentences they think in Chinese grammar.

\section{(b): Structure confusion}

As the sentences are written according to the native language structure in the students' mind, many sentences are wrong in their structure. Therefore many sentences with structural confusion can be found. My students wrote in their homework: "In a word creatures is not living without it" (Wrong use of predicate. It means "In a word, all creatures cannot live without it".); "In March and November has a slightly higher temperature." (Incorrect use of subject and predicate: It means "In March and November the temperature is slightly higher than usually"). "There is different, we need every kind of air is different too" (The structure of the whole sentence is wrong. It means "The gases in the air are different in quantity and the amount of every gas we need is different, too"); "Air is important for us to live, study and so on. We should protect our air in order to we find a better environment to study and work" (Incorrect use of adverbial infinitive phrase, It means "As air is important for our life, study and work. We should protect it from being polluted so that we can have a better environment"); "But it is the most hot in city A than in city B" (Incorrect use of comparison. It means: "But as compared with the temperature in city B, city A is hotter"); "D and E are not as same the size as them" (Wrong use of predictive and adverbial, It means: "Bottle D and Bottle E are not the same in size as other bottles"); There are many other such structural confusion sentences in the students homework. The above are just a few examples among many. As they are too many to list the authors will not list them one by one.

\section{(c): Incorrect use of words}

Sometimes the students, if not well trained, will most likely write English articles in sentences piled with English words. For example, there are some sentences in my students' homework: "It's containing is four times as much as Bottle B and Bottle A" (Meaning: The amount of liquid it contains is four times as much as that in Bottle A). "On July the temperature of the two cities begin to reduce." (Meaning: "In July the temperature of the two cities begins to fall"). The students do not use the words correctly.

\section{(d): Grammatically wrong in the whole sentence}

According to the authors' investigation, many college students may write such Chinese Tone English "Chintonglish" sentences in their written homework. For example: "Its contain is 12 Liters". (Meaning: It contains 12 liters). "Bottle A contains red ink least" (Meaning: "The liquid contained in Bottle A is the least" or "Bottle A contains least liquid"); "We also can know the high temperature months in Nanchang is more than in Beijing" (Meaning: "We can also know that the temperature in Nanchang during the hotter months is higher than that in Beijing"); "Any living thing all must depend on air to live through" (Meaning: "All living things depend on the air for their living"). Therefore the following stereotyped pattern appears:

我（已）收到你的信三个星期（了）

$\downarrow \quad \downarrow \quad \downarrow \quad \downarrow$

I have received your letter for three weeks.

Look every part of the sentence is in strict conformity with the structure of Chinese language. While writing this English letter, the student has nothing in his mind but some English words or expressions, with the result that the sentence he wrote becomes a sentence with broken grammar.

\section{(C) The cause behind such Chinese Tone English "Chintonglish" sentences}

Writing is a complicated psychological process, which involves many factors. Besides, English writing involves many other factors too. It involves not only the writer's English level, but his cultural background as well. This is why English writing has always been one of the headache problems for Chinese students. Up to now we are in the dark as to the concrete psychological process in the students mind in their English writing. No matter how well the students' English may be, as the students live and work in a Chinese cultural surrounding, they must eventually be influenced by their Chinese culture. No matter how high a man jump he will eventually fall to the same place he is in. Therefore the problem is how to reduce the influence of the Chinese cultural impact on the students' mind to the minimum in their 
English writing.

However, no matter how hard a student may try to write his English well he may not get rid of this cultural impact completely on his mind in every process of writing. Let's brush aside the legends with traditional Chinese cultural background, so far as ordinary sentence is concerned, the Chinese students may most likely to be influenced by the structure of his native language --- Chinese --- sentence structure, resulting in a Chinese Tone English "Chintonglish" sentences.

Take the sentence at the beginning of this paper for example: "I have received your letter for three weeks". Obviously this sentence means “我收到你的信已经三个星期了”. In the eye of a foreigner it is a wrong sentence according to his own native language pattern. The process is as follows: In writing this letter the student, first of all, thinks in Chinese. Thus the Chinese sentence “我收到你的信已经三个星期了” appears in his mind . Then he managed to "Translate" it into "English" word by word, i.e. according to his native language structure. In the eye of the student, by translation or writing refer just the change of every Chinese expression into English, ignoring its English character, to say nothing of the textural structure. The following sentences are some typical examples:

1 In summer welcome to Nanchang

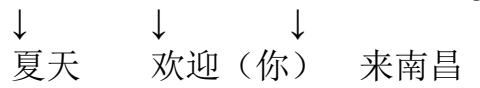

2 Any living thing all must depend on air to live through.

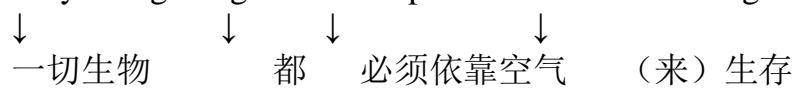

It can be seen that all the above examples are nothing but Chinese Tone English "Chintonglish" sentences. No matter how hard a teacher may try to do so, this type of "English" sentences can not be completely eliminated, for the most famous translators cannot completely avoid such sentences, to say nothing of English beginners. The teacher must point out where lies the mistake in front of all the students so that the students may try their best to avoid such mistakes as much as possible.

\section{(2) Create an English communicative environment}

As the Chinese students are "surrounded" by their Chinese cultural environment all the times, they must unavoidably be influenced by their native language and culture in their writing. Therefore the most important thing is to create an English atmosphere for the students so that they can have a "oasis" to live in. To the English-thirsty students this "oasis" is of great importance for their English study. Different from foreign students, who are grown up in foreign atmosphere, Chinese students are grown up in Chinese cultural environment, every action and every word bear the brand of Chinese culture and language. Chinese Tone English "Chintonglish" is a piece of writing, which is just a collection of English words only sometimes here and there with some idiomatic expressions. It is just like a Chinese actor dressed in foreign costume. No matter how hard he may act he will not act like a foreigner does. So the teacher's task is a try to create an English atmosphere whenever possible. For example, conduct a communicative teaching course in the class, hold an English "corner" in school campus or in street corner, or in parks etc. where the students can talk with foreigners at will and on any topic they like. This is a very good way for the students to enrich their English knowledge and enlarge their vocabulary.

(3) Choose some typical pieces of passages for the students to read and imitate.

Reading and imitation have always been two ideal ways for students to learn typical English. The merits of reading and imitation of some typical pieces of English writings is that the students can imitate not only the great writers' ability to control the greatest invention man has ever created --- the language, but their power to use the most elusive phenomenon of language --- the style, so that they can naturally write perfect English. It is well known that in both the history of East and west, many famous writers have benefited much from reading and imitating famous master's writings. For example, famous British writer R.L. Stevenson had benefited greatly from his reading and imitation of famous writings. He said: Whenever I read a book or a passage that particularly pleased me, in which a thing was said or an effect rendered with propriety in which there was either some conspicuous force or some distinction in the style, I must sit down at once and set myself to ape that quality. As teachers we must choose some typical pieces of passages for the students to read and imitate.

\section{SUMMARY}

The Chinese Tone English (Chintonglish) has no communicative value. It is generally not accepted in communication for they are considered as wrong sentences. It is an unavoidable process in the students' written homework. Therefore, the teacher's task is to try to "eliminate" such kind of Chinese Tone English (Chintonglish) sentences in their writing, so that the students may be as close to the foreigner's writing as possible.

The Chinese Tone English (Chintonglish) are typical Chinese in nature both in the grammar or in the expressions. They are completely broken English in the eye of a foreigner. Chinese Tone English (Chintonglish) exists among the students in great number and the examples are too many to list, the author thinks it is necessary to conduct a deep-going research into this type of English in great details so as to find its real causes. We should find the methods used for the elimination of such Chintonglish sentences in the students' English writing. This is the chief purpose for the writing of 
this paper.

\section{REFERENCES}

[1] Cai Yun. (1991). "Writing Process and Writing Ability". Modern Foreign Languages. Guangzhou. (4): $52-59$

[2] Catherine Ann Cameron, King Lee, Suzanne Webster, Kim Munro, Anne Kalthryn Hunt, Muttay J. Linton. (1995). "Text Cohesion in Children's Narrative (1996) Writing”. Applied Psycholinguistics. Cambridge University Press. Britain.

[3] Chen Jinying. (1994). "How to Teach Chinese Students to Write Good English" Foreign Language Teaching and Research, Beijing, (2): 21-25

[4] Cai Yun. (1991). "Writing Process and Writing Ability". Moden Foreign Languages. Guangzhou. (4): 53-59

[5] Huang Weixin. (1996). "A Case Study: Current Problems in English Students' Reading and Writing”. Modern Foreign Languages. Guangzhou. (9): 46-51

[6] Li Wenzhong. (1993). “China English and Chinglish” Foreign Language Teaching and Research, Beijing, (4): 34-39

[7] Lin Li. (1991). "Analysis of the Teaching of College English Writing”. Modern Foreign Languages. Guangzhou. (7): 54-60

[8] Xie Zhijun. (1995). China English: Interference Variety in Cross culture Cummunication. Modern Foreign Languages. Guangzhou. (2) 54-60.

[9] Xu Liejiong. (1994). The Width and Depth of Cross-linguistic studies. Foreign Languages Teaching and Research, Beijing. (9): 64-70.

[10] Yang Zhonglin. (1994). The Role of proofreading in English Proficiency Test. Foreign Languages Teaching and Research, Beijing (11): 47-56.

[11] Yang Yuchen \& Wen Zaorong. (1994). "An Analysis on Sentence Types and Structures in Chinese Students' English Writing”. Modern Foreign Languages. Guangzhou. (5): 28-33.

[12] Zhao Yongqing. (1995). From Written Discourse to Teaching of Reading. Modern Foreign Languages. Guangzhou. (1) 36-41

[13] Zhang Zuochen. (1995). An Account of the Psychological Process of Writing for Chinese Learners of English. Modern Foreign Languages. Guangzhou. (10) 62-69

[14] Zhang Jin. (1981). "An Outline of Comparative Grammar between English and Chinese" commercial Press, Beijing, PRC.

Xiaoying Zhou was born in Nanchang, China in 1962. She received her Bachelor of Arts in Foreign Language Department in Huadong Normal University, Shanghai, China in 1987.

She is currently a professor in the School of Foreign Languages, Nanchang Normal University, Nanchang, China. Her research interests include linguistics and English teaching methods.

Hangjie Liao was born in Yichun, China in 1989. He received his M.S degree in Aeronautics and Astronautics Engineering from Purdue University, USA, in 2012. He is currently pursuing the PH.D degree in Purdue University.

$\mathrm{He}$ is a Research Assistant in Purdue University. 